\title{
Synthesis and characterization of 5-heteroarylsulfanyl-4-aryl- 1,2,3-selena/thiadiazoles
}

\author{
RAMAIYAN MANIKANNAN, MASILAMANI SHANMUGARAJA, \\ SEETHARAMAN MANOJVEER and SHANMUGAM MUTHUSUBRAMANIAN* \\ Department of Organic Chemistry, Madurai Kamaraj University, Madurai 625021, India \\ e-mail: muthumanian2001@yahoo.com
}

MS received 9 May 2011; revised 12 July 2011; accepted 22 July 2011

\begin{abstract}
Synthesis and spectral characterization of 2-methyl-5-[(4-aryl-1,2,3-selenadiazol-5-yl)sulfanyl]1,3,4-thiadiazoles, 5-[4-aryl-1,2,3-selenadiazol-5-yl]sulfanyl-1-phenyl-1 $H$-1,2,3,4-tetraazoles, 4-aryl-5-[(5methyl-1,3,4-thiadiazol-2-yl)sulfanyl]-1,2,3-thiadiazole and 5-[4-aryl-1,2,3-thiadiazol-5-yl]sulfanyl-1-phenyl$1 H-1,2,3,4$-tetraazole have been reported.
\end{abstract}

Keywords. 1,2,3-Selenadiazoles; 1,2,3-thiadiazoles; 5-methyl-1,3,4-thiadiazole; 1-phenyl-1 $H$-tetrazole; diheteroaryl sulfide.

\section{Introduction}

1,3,4-Thiadiazole derivatives have potent pharmacological activities like antibacterial, ${ }^{1}$ antifungal, ${ }^{2}$ antinflammatory, ${ }^{3}$ herbicidal, ${ }^{4}$ inhibitory activity, ${ }^{5}$ analgesic activity, ${ }^{6}$ antihistaminic and anticholinergic, ${ }^{7}$ plantgrowth regulating activity etc. ${ }^{8}$ Compounds with a 1,2,3-thiadiazole moiety have been found to exhibit various pharmacological properties like antifungal, ${ }^{9}$ pesticidal $^{10}$ and antibacterial activities. 4,5-Bis(pmethoxyphenyl)-1,2,3-thiadiazole possesses platelet aggregation inhibitory activity in humans ${ }^{11}$ and some 1,2,3-thiadiazole derivatives are useful for the therapeutic and prophylactic treatment of viral, bacterial, fungal, and parasitic infections in humans and animals. ${ }^{12}$

Pentamethylene tetrazole is used as a stimulant for the central nervous system and to counteract the effects of over dosage of barbiturates. ${ }^{13}$ Anti-inflammatory activity is shown by both substituted and unsubstituted tetrazoles. ${ }^{14}$ A range of cephalosporin derivatives containing substituted tetrazole rings has been prepared and found to display antibacterial activity. An active antilipemic tetrazole molecule has progressed to clinical tests. ${ }^{15}$ A large number of substituted chromone derivatives with tetrazole groups located at C-2 and C-3 have been prepared as potential antiallergic agents.

Compounds containing 1,2,3-selenadiazole moieties are well-known for their pharmacological properties like antifungal ${ }^{16}$ and antibacterial activities. ${ }^{17}$ Antibacterial activities of selenadiazoles fused with

*For correspondence cycloheptindoles, ${ }^{18}$ pyridines $^{19}$ and 5,7-diphenyl(4alkyl)trihydropyran ${ }^{20}$ have also been investigated. 1,2, 3-Selenadiazoles bearing benzene-sulfonamides at 4position have been tested for their antibacterial activity and most of them showed strong activity against Bacillus cereus and moderate activity against Escherichia choli. ${ }^{21,22}$ 1,2,3-Selenadiazoles have been effectively utilized in the synthesis of semiconductor nanoparticles or nanopowder. ${ }^{23}$

\section{Experimental}

\subsection{Materials, methods and instruments}

Melting points were determined in open capillaries and are uncorrected. NMR spectra were recorded on a Bruker $300 \mathrm{MHz}$ instrument in DMSO- $\mathrm{d}_{6} / \mathrm{CDCl}_{3}$ using TMS as internal standard. Chemical shifts are given in parts per million ( $\delta$-scale) and coupling constants are given in Hertz. NMR spectra are provided in the Supporting Information section. Microanalyses were carried out on a Perkin-Elmer instrument. Column chromatography was carried out in silica gel (60-120 mesh) using pet ether-ethyl acetate as eluent.

\subsection{The precursors for the semicarbazones $\mathbf{4 a - c}$}

2.2a 1-Phenyl-2-[(1-phenyl-1H-1,2,3,4-tetraazol-5$y$ l)sulfanyl]-1-ethanone: $94 \%$ yield, m.p. $112^{\circ} \mathrm{C}$, time 10 min. ${ }^{1} \mathrm{H}$ NMR $\left(300 \mathrm{MHz}, \mathrm{CDCl}_{3}\right): \delta 5.10(\mathrm{~s}, 2 \mathrm{H}$, 
$\left.-\mathrm{CH}_{2}\right), 7.55-7.67(\mathrm{~m}, 8 \mathrm{H},-\mathrm{Ar}-H), 8.05(\mathrm{~d}, J=7.5 \mathrm{~Hz}$, $2 \mathrm{H},-\mathrm{Ar}-H) ;{ }^{13} \mathrm{C}$ NMR $\left(75 \mathrm{MHz}, \mathrm{CDCl}_{3}\right): \delta 42.6$, 123.7, 128.5, 128.9, 129.9, 130.3, 133.4, 134.3, 134.8, 153.5, 192.1. Anal. Calcd. for $\mathrm{C}_{15} \mathrm{H}_{12} \mathrm{~N}_{4} \mathrm{OS}: \mathrm{C} 60.79 \mathrm{H}$ 4.08 N 18.91. Found: C 60.91 H 4.16 N 19.02.

2.2b 1-(4-Nitrophenyl)-2-[(1-phenyl-1H-1,2,3,4tetraazol-5-yl)sulfanyl]-1-ethanone: $93 \%$ yield, m.p. $173-174^{\circ} \mathrm{C}$, time $10 \mathrm{~min} .{ }^{1} \mathrm{H}$ NMR $(300 \mathrm{MHz}$, DMSO$\left.\mathrm{d}_{6}\right): \delta 5.19\left(\mathrm{~s}, 2 \mathrm{H},-\mathrm{CH}_{2}\right), 7.67-8.38(\mathrm{~m}, 10 \mathrm{H},-\mathrm{Ar}-H)$; ${ }^{13} \mathrm{C}$ NMR (75 MHz, DMSO-d 6 ): $\delta 41.8,124.0^{*}, 130.0$, 130.6, 133.5, 140.1, 150.2, 153.4, 192.4. Anal. Calcd. for $\mathrm{C}_{15} \mathrm{H}_{11} \mathrm{~N}_{5} \mathrm{O}_{3} \mathrm{~S}$ : C $52.78 \mathrm{H} 3.25 \mathrm{~N}$ 20.52. Found: $\mathrm{C}$ 52.84 H 3.34 N 20.67.

2.2c 1-(4-Chlorophenyl)-2-[(1-phenyl-1H-1,2,3,4tetraazol-5-yl)sulfanyl]-1-ethanone: $94 \%$ yield, m.p. $178^{\circ} \mathrm{C}$, time $10 \mathrm{~min} .{ }^{1} \mathrm{H}$ NMR $\left(300 \mathrm{MHz}, \mathrm{CDCl}_{3}\right): \delta$ $5.04\left(\mathrm{~s}, 2 \mathrm{H},-\mathrm{CH}_{2}\right), 7.50(\mathrm{~d}, J=8.4 \mathrm{~Hz}, 2 \mathrm{H},-\operatorname{Ar}-H)$, 7.56-7.63 (m, 5H, -Ar- $H$ ), $8.00(\mathrm{~d}, J=8.7 \mathrm{~Hz}, 2 \mathrm{H}$, -Ar- $H) ;{ }^{13} \mathrm{C}$ NMR $\left(75 \mathrm{MHz}, \mathrm{CDCl}_{3}\right): \delta 42.3,123.8$, 129.4, 129.9, 130.0, 130.3, 133.2, 133.4, 140.9, 153.3, 191.0. Anal. Calcd. for $\mathrm{C}_{15} \mathrm{H}_{11} \mathrm{ClN}_{4} \mathrm{OS}$ : C $54.46 \mathrm{H} 3.35$ $\mathrm{N}$ 16.94. Found: C $54.58 \mathrm{H} 3.43 \mathrm{~N} 17.02$, are new and have been prepared by the methods adopted for the other ketones.

\subsection{General procedure for the preparation of semicarbazones $\mathbf{2 a}-\mathbf{f}$ and $\mathbf{4 a - c}$}

To a warm ethanolic solution of 2-(5-methyl-1,3,4-thiadiazolesulfanyl)-1-aryl-1-ethanone/1-aryl-2-[(1-phenyl-1 $H$ 1,2,3,4-tetraazol-5-yl)sulfanyl]-1-ethanone (0.005 mol), aqueous solution of semicarbazide hydrochloride $(0.035 \mathrm{~mol})$ and sodium acetate $(0.035 \mathrm{~mol})$ was added carefully avoiding turbidity formation. The reaction mixture was refluxed for 1.5-2 h, poured onto crushed ice and filtered. Semicarbazones $\mathbf{2}$ were crystallized from ethanol and semicarbazones $\mathbf{4}$, obtained as viscous liquid, have not been characterized due to their poor solubility in NMR solvents and used as such for next stage.

2.3a 2-[(5-Methyl-1,3,4-thiadiazol-2-yl)sulfanyl]-1phenylethylidene-1-hydrazine carboxamide (2a): $90 \%$ yield, m.p. $115-116^{\circ} \mathrm{C}$, time 1.5 h. ${ }^{1} \mathrm{H}$ NMR $\left(300 \mathrm{MHz}, \mathrm{CDCl}_{3}\right): \delta 2.71\left(\mathrm{~s}, 3 \mathrm{H},-\mathrm{CH}_{3}\right), 4.57$ (s, $2 \mathrm{H},-\mathrm{CH}_{2}$ ), 5.02-6.30 (bs, $\left.2 \mathrm{H}, \mathrm{NH}_{2}\right), 7.26-7.68$ (m, $5 \mathrm{H},-\operatorname{Ar} H), 10.21(\mathrm{~s}, 1 \mathrm{H},-\mathrm{NH}) ;{ }^{13} \mathrm{C}$ NMR $(75 \mathrm{MHz}$, $\left.\mathrm{CDCl}_{3}\right): \delta 15.7,27.3,127.3,128.6,129.5,135.8,142.7$,
157.7, 164.4, 165.0. Anal. Calcd. for $\mathrm{C}_{12} \mathrm{H}_{13} \mathrm{~N}_{5} \mathrm{OS}_{2}$ : C 46.89 H 4.26 N 22.78. Found: C 46.98 H 4.36 N 22.86.

2.3b 1-(4-Methylphenyl)-2-[(5-methyl-1,3,4-thiadiazol2-yl)sulfanyl]ethylidene-1-hydrazine carboxamide (2b): $90 \%$ yield, m.p. $125-126^{\circ} \mathrm{C}$, time 1.5 h. ${ }^{1} \mathrm{H}$ NMR $\left(300 \mathrm{MHz}, \mathrm{CDCl}_{3}\right): \delta 2.39\left(\mathrm{~s}, 3 \mathrm{H},-\mathrm{CH}_{3}\right), 2.74(\mathrm{~s}$, $\left.3 \mathrm{H},-\mathrm{CH}_{3}\right), 4.56\left(\mathrm{~s}, 2 \mathrm{H},-\mathrm{CH}_{2}\right), 5.02-6.30(\mathrm{bs}, 2 \mathrm{H}$, $\left.-\mathrm{NH}_{2}\right), 7.22(\mathrm{~d}, J=8.1 \mathrm{~Hz}, 2 \mathrm{H},-\mathrm{Ar} H), 7.57(\mathrm{~d}, J=$ $8.1 \mathrm{~Hz}, 2 \mathrm{H},-\mathrm{Ar} H), 10.13(\mathrm{~s}, 1 \mathrm{H},-\mathrm{NH}) ;{ }^{13} \mathrm{C} \mathrm{NMR}$ $\left(75 \mathrm{MHz}, \mathrm{CDCl}_{3}\right): \delta 15.7,21.3,27.1,125.9,129.4$, 133.0, 139.8, 143.0, 157.7, 164.4, 165.9. Anal. Calcd. for $\mathrm{C}_{13} \mathrm{H}_{15} \mathrm{~N}_{5} \mathrm{OS}_{2}$ : C $48.58 \mathrm{H} 4.70 \mathrm{~N} 21.79$. Found: $\mathrm{C}$ $48.64 \mathrm{H} 4.83$ N 21.92.

2.3c 1-(4-Methoxyphenyl)-2-[(5-methyl-1,3,4-thiadiazol-2-yl)sulfanyl]ethylidene-1-hydrazinecarboxamide (2c): $91 \%$ yield, m.p. $120-121{ }^{\circ} \mathrm{C}$, time 1.5 h. ${ }^{1} \mathrm{H}$ $\mathrm{NMR}\left(300 \mathrm{MHz}, \mathrm{CDCl}_{3}\right): \delta 2.73\left(\mathrm{~s}, 3 \mathrm{H},-\mathrm{CH}_{3}\right), 3.85$ (s, 3H, - $\left.\mathrm{OCH}_{3}\right), 4.55$ (s, 2H, - $\left.\mathrm{CH}_{2}\right), 5.10-6.40$ (bs, 2H, $\left.\mathrm{NH}_{2}\right), 6.92(\mathrm{~d}, J=8.7 \mathrm{~Hz}, 2 \mathrm{H},-\operatorname{Ar} H), 7.64(\mathrm{~d}, J=$ $8.7 \mathrm{~Hz}, 2 \mathrm{H},-\mathrm{Ar} H), 10.05(\mathrm{~s}, 1 \mathrm{H},-\mathrm{NH}) ;{ }^{13} \mathrm{C} \mathrm{NMR}$ $\left(75 \mathrm{MHz}, \mathrm{CDCl}_{3}\right): \delta 15.7,27.2,55.3,114.0,127.5$, 128.3, 142.7, 157.7, 160.8, 164.4, 165.9. Anal. Calcd. for $\mathrm{C}_{13} \mathrm{H}_{15} \mathrm{~N}_{5} \mathrm{O}_{2} \mathrm{~S}_{2}$ : C $46.27 \mathrm{H} 4.48 \mathrm{~N} 20.76$. Found: $\mathrm{C}$ $46.38 \mathrm{H} 4.54 \mathrm{~N} 20.87$.

2.3d 1-(4-Chlorophenyl)-2-[(5-methyl-1,3,4-thiadiazol-2-yl)sulfanyl]ethylidene-1-hydrazinecarboxamide (2d): $94 \%$ yiled, m.p. $148-149^{\circ} \mathrm{C}$, time 1.5 h. ${ }^{1} \mathrm{H}$ NMR $\left(300 \mathrm{MHz}, \mathrm{CDCl}_{3}\right): \delta 2.72\left(\mathrm{~s}, 3 \mathrm{H},-\mathrm{CH}_{3}\right), 4.57$ (s, 2H, $\left.-\mathrm{CH}_{2}\right), 5.12-6.40$ (bs, 2H, $\left.-\mathrm{NH}_{2}\right), 7.36(\mathrm{~d}, J=$ $8.4 \mathrm{~Hz}, 2 \mathrm{H},-\operatorname{Ar} H), 7.62(\mathrm{~d}, J=8.4 \mathrm{~Hz}, 2 \mathrm{H},-\operatorname{Ar} H)$, $10.29(\mathrm{~s}, 1 \mathrm{H},-\mathrm{NH}) ;{ }^{13} \mathrm{C} \mathrm{NMR}\left(75 \mathrm{MHz}, \mathrm{CDCl}_{3}\right): \delta$ 15.7, 27.3, 127.3, 128.8, 134.4, 135.4, 141.7, 157.7, 165.0, 166.1. Anal. Calcd. for $\mathrm{C}_{12} \mathrm{H}_{12} \mathrm{ClN}_{5} \mathrm{OS}_{2}$ : C 42.16 H 3.54 N 20.49. Found: C 42.24 H 3.62 N 20.56.

$2.3 \mathrm{e} \quad 2-[(5-M e t h y l-1,3,4-$ thiadiazol-2-yl)sulfanyl]-1(4-nitrophenyl)ethylidene]-1-hydrazine carboxamide (2e): $97 \%$ yield, m.p. $110-111^{\circ} \mathrm{C}$, time 1.5 h. ${ }^{1} \mathrm{H}$ $\mathrm{NMR}\left(300 \mathrm{MHz}, \mathrm{CDCl}_{3}\right): \delta 2.74\left(\mathrm{~s}, 3 \mathrm{H},-\mathrm{CH}_{3}\right), 4.62$ (s, 2H, - $\left.\mathrm{CH}_{2}\right), 5.12-6.40$ (bs, 2H, $\left.-\mathrm{NH}_{2}\right), 7.87$ (d, $J=$ $9.0 \mathrm{~Hz}, 2 \mathrm{H},-\operatorname{Ar}-H), 8.27$ (d, $J=9.0 \mathrm{~Hz}, 2 \mathrm{H},-\operatorname{Ar}-H)$; $10.55(\mathrm{~s}, 1 \mathrm{H},-\mathrm{NH}) ;{ }^{13} \mathrm{C}$ NMR $\left(75 \mathrm{MHz}, \mathrm{CDCl}_{3}\right): \delta$ $15.8,27.4,123.9,126.7,129.8,130.1,142.1,157.9$, 159.7, 160.3. Anal. Calcd. for $\mathrm{C}_{12} \mathrm{H}_{12} \mathrm{~N}_{6} \mathrm{O}_{3} \mathrm{~S}_{2}$ : C 40.90 H 3.43 N 23.85. Found: C 40.98 H 3.52 N 23.94. 
2.3f 2-[(5-Methyl-1,3,4-thiadiazol-2-yl)sulfanyl]-1-(2naphthyl)ethylidene]-1-hydrazine carboxamide (2f): $93 \%$ yield, m.p. $132-133^{\circ} \mathrm{C}$, time 2 h. ${ }^{1} \mathrm{H}$ NMR $\left(300 \mathrm{MHz}, \mathrm{CDCl}_{3}\right): \delta 2.72\left(\mathrm{~s}, 3 \mathrm{H},-\mathrm{CH}_{3}\right), 4.69$ (s, 2H, $-\mathrm{CH}_{2}$ ), 5.12-6.40 (bs, 2H, $\left.-\mathrm{NH}_{2}\right), 7.52-8.08$ (m, 6H, Ar- $H), 10.26$ (s, $2 \mathrm{H},-\mathrm{Ar} H,-\mathrm{NH}) ;{ }^{13} \mathrm{C}$ NMR $(75 \mathrm{MHz}$, $\left.\mathrm{CDCl}_{3}\right): \delta 15.6,27.1,123.2,125.7,126.6,127.8,128.4$, 128.7, 129.7, 132.3, 131.1, 133.6, 142.7, 164.3, 165.4, 166.0. Anal. Calcd. for $\mathrm{C}_{16} \mathrm{H}_{15} \mathrm{~N}_{5} \mathrm{OS}_{2}$ : C $53.76 \mathrm{H} 4.23$ N 19.59. Found: C 53.87 H 4.32 N 19.67.

\subsection{General procedure for the preparation of 1,2,3-selenadiazole derivatives $\mathbf{3 a - f}$ and $\mathbf{5 a}-\mathbf{b}$}

A solution of $(0.001 \mathrm{~mol})$ of the appropriate semicarbazones $\mathbf{2}$ or $\mathbf{4}$ was dissolved in dry THF by gentle warming and $(0.005 \mathrm{~mol})$ of powdered selenium dioxide was added by portion. The reaction mixture was heated to reflux on a water bath for an hour. The selenium deposited on cooling was removed by filtration and the filtrate was poured into crushed ice and extracted with chloroform. The product was obtained upon purification by column chromatography using silica gel (60-120 mesh).

2.4a 2-Methyl-5-[(4-phenyl-1,2,3-selenadiazol-5yl)sulfanyl]-1,3,4-thiadiazole (3a): $74 \%$ yield, m.p. 130-131 ${ }^{\circ} \mathrm{C} .{ }^{1} \mathrm{H}$ NMR $\left(300 \mathrm{MHz}, \mathrm{CDCl}_{3}\right): \delta 2.82(\mathrm{~s}$, $\left.3 \mathrm{H},-\mathrm{CH}_{3}\right), 7.60(\mathrm{~m}, 3 \mathrm{H},-\mathrm{Ar}-H), 7.84(\mathrm{~d}, J=7.5 \mathrm{~Hz}$, $2 \mathrm{H},-\mathrm{Ar}-H) ;{ }^{13} \mathrm{C} \mathrm{NMR}\left(75 \mathrm{MHz}, \mathrm{CDCl}_{3}\right): \delta 16.0$, 129.1, 129.2, 129.3, 131.7, 142.1, 158.4, 159.2, 167.9. Anal. Calcd. for $\mathrm{C}_{11} \mathrm{H}_{8} \mathrm{~N}_{4} \mathrm{~S}_{2} \mathrm{Se}$ : C $38.94 \mathrm{H} 2.38 \mathrm{~N}$ 16.51. Found: C 39.05 H 2.48 N 16.65.

2.4b 2-Methyl-5-[4-(4-methylphenyl)-1,2,3-selenadiazol-5-yl]sulfanyl-1,3,4-thiadiazole (3b): $70 \%$ yield, m.p. $131-132^{\circ} \mathrm{C} .{ }^{1} \mathrm{H}$ NMR $\left(300 \mathrm{MHz}, \mathrm{CDCl}_{3}\right): \delta 2.45$ $\left(\mathrm{s}, 3 \mathrm{H},-\mathrm{CH}_{3}\right), 2.81\left(\mathrm{~s}, 3 \mathrm{H},-\mathrm{CH}_{3}\right), 7.36(\mathrm{~d}, J=8.0 \mathrm{~Hz}$, $2 \mathrm{H},-\operatorname{Ar}-H), 7.72(\mathrm{~d}, J=8.0 \mathrm{~Hz}, 2 \mathrm{H},-\operatorname{Ar}-H) ;{ }^{13} \mathrm{C}$ NMR $\left(75 \mathrm{MHz}, \mathrm{CDCl}_{3}\right): \delta 16.0,21.4,128.8,129.1$, 129.6, 139.4, 141.2, 158.5, 159.7, 167.8. Anal. Calcd. for $\mathrm{C}_{12} \mathrm{H}_{10} \mathrm{~N}_{4} \mathrm{~S}_{2} \mathrm{Se}$ : C $40.79 \mathrm{H} 2.85 \mathrm{~N}$ 15.86. Found: C 40.86 H 2.92 N 15.92 .

2.4c 2-[4-(4-Methoxyphenyl)-1,2,3-selenadiazol-5yl]sulfanyl-5-methyl-1,3,4-thiadiazole (3c): $72 \%$ yield, m.p. ${ }^{135}-136^{\circ} \mathrm{C} .{ }^{1} \mathrm{H} \mathrm{NMR}\left(300 \mathrm{MHz}, \mathrm{CDCl}_{3}\right): \delta 2.69$ $\left(\mathrm{s}, 3 \mathrm{H},-\mathrm{CH}_{3}\right), 3.77\left(\mathrm{~s}, 3 \mathrm{H},-\mathrm{OCH}_{3}\right), 6.96(\mathrm{~d}, J=$ $8.9 \mathrm{~Hz}, 2 \mathrm{H},-\mathrm{Ar}-H), 7.66(\mathrm{~d}, J=8.9 \mathrm{~Hz}, 2 \mathrm{H},-\mathrm{Ar}-H)$; ${ }^{13} \mathrm{C} \mathrm{NMR}\left(75 \mathrm{MHz}, \mathrm{CDCl}_{3}\right): \delta 16.0,55.4,114.3,124.1$,
130.6, 140.6, 158.3, 159.8, 160.3, 167.8. Anal. Calcd. for $\mathrm{C}_{12} \mathrm{H}_{10} \mathrm{~N}_{4} \mathrm{OS}_{2} \mathrm{Se}$ : C $39.02 \mathrm{H} 2.73 \mathrm{~N}$ 15.17. Found: C 39.11 H 2.82 N 15.25 .

2.4d 2-[4-(4-Chlorophenyl)-1,2,3-selenadiazol-5yl]sulfanyl-5-methyl-1,3,4-thiadiazole (3d): $69 \%$ yield, m.p. $145-146^{\circ} \mathrm{C} .{ }^{1} \mathrm{H}$ NMR $\left(300 \mathrm{MHz}, \mathrm{CDCl}_{3}\right): \delta 2.86$ $\left(\mathrm{s}, 3 \mathrm{H},-\mathrm{CH}_{3}\right), 7.56(\mathrm{~d}, J=8.3 \mathrm{~Hz}, 2 \mathrm{H},-\mathrm{Ar}-H), 7.83$ $(\mathrm{d}, J=8.3 \mathrm{~Hz}, 2 \mathrm{H},-\mathrm{Ar}-H) ;{ }^{13} \mathrm{C} \mathrm{NMR}(75 \mathrm{MHz}$, $\left.\mathrm{CDCl}_{3}\right): \delta 16.1,129.2,129.4,131.2,141.8,158.6$, 159.3, 163.0, 167.8. Anal. Calcd. for $\mathrm{C}_{11} \mathrm{H}_{7} \mathrm{ClN}_{4} \mathrm{~S}_{2} \mathrm{Se}$ : C 35.35 H 1.89 N 14.99. Found: C 35.46 H 1.96 N 15.09 .

2.4e 2-Methyl-5-[4-(4-nitrophenyl)-1,2,3-selenadiazol-5-yl]sulfanyl-1,3,4-thiadiazole (3e): $79 \%$ yield, m.p. $125-126^{\circ} \mathrm{C} .{ }^{1} \mathrm{H}$ NMR $\left(300 \mathrm{MHz}, \mathrm{CDCl}_{3}\right): \delta 2.86$ (s, $\left.3 \mathrm{H},-\mathrm{CH}_{3}\right), 8.12$ (d, $\left.J=8.9 \mathrm{~Hz}, 2 \mathrm{H},-\mathrm{Ar}-H\right), 8.43$ $(\mathrm{d}, J=8.9 \mathrm{~Hz}, 2 \mathrm{H},-\mathrm{Ar}-H) ;{ }^{13} \mathrm{C} \mathrm{NMR}(75 \mathrm{MHz}$, $\left.\mathrm{CDCl}_{3}\right): \delta 16.0,129.0,129.2,129.3,131.7,141.7$, 158.3, 159.6, 167.9. Anal. Calcd. for $\mathrm{C}_{11} \mathrm{H}_{7} \mathrm{~N}_{5} \mathrm{O}_{2} \mathrm{~S}_{2} \mathrm{Se}$ : C 34.38 H 1.84 N 18.22. Found: C 34.46 H $1.91 \mathrm{~N}$ 18.33 .

2.4f 2-Methyl-5-[4-(2-naphthyl)-1,2,3-selenadiazol5-yl]sulfanyl-1,3,4-thiadiazole (3f): $76 \%$ yield, m.p. $140-141^{\circ} \mathrm{C}$, time 1 h. ${ }^{1} \mathrm{H}$ NMR $\left(300 \mathrm{MHz}, \mathrm{CDCl}_{3}\right): \delta$ $2.82\left(\mathrm{~s}, 3 \mathrm{H},-\mathrm{CH}_{3}\right), 7.52-8.31(\mathrm{~m}, 9 \mathrm{H},-\mathrm{Ar}-\mathrm{H}), 8.59$ $(\mathrm{s}, 1 \mathrm{H},-\mathrm{Ar}-H) ;{ }^{13} \mathrm{C}$ NMR $\left(75 \mathrm{MHz}, \mathrm{CDCl}_{3}\right): \delta 16.0$, 126.5, 126.7, 127.1, 127.8, 128.4, 128.8, 129.2, 130.7, 133.1, 133.4, 142.0, 158.3, 159.6, 167.9. Anal. Calcd. for $\mathrm{C}_{15} \mathrm{H}_{10} \mathrm{~N}_{4} \mathrm{~S}_{2} \mathrm{Se}$ : C $46.27 \mathrm{H} 2.59 \mathrm{~N}$ 14.39. Found: C $46.34 \mathrm{H} 2.65 \mathrm{~N} 14.45$.

2.4g 5-[4-Phenyl-1,2,3-selenadiazol-5-yl]sulfanyl-1phenyl-1H-1,2,3,4-tetraazole (5a): $65 \%$ yield, m.p. $152-153^{\circ} \mathrm{C} .{ }^{1} \mathrm{H}$ NMR $\left(300 \mathrm{MHz}, \mathrm{CDCl}_{3}\right): \delta 7.32-7.70$ $(\mathrm{m}, 10 \mathrm{H},-\mathrm{Ar}-H) ;{ }^{13} \mathrm{C} \mathrm{NMR}\left(75 \mathrm{MHz}, \mathrm{CDCl}_{3}\right): \delta 123.9$, 129.0, 129.2, 129.5, 129.8, 130.2, 131.1, 132.7, 140.3, 150.2, 159.4. Anal. Calcd. for $\mathrm{C}_{15} \mathrm{H}_{10} \mathrm{~N}_{6} \mathrm{SSe}$ : C 46.76 H 2.62 N 21.81. Found: C 46.87 H 2.78 N 21.94.

2.4h 5-[4-(4-Nitrophenyl)-1,2,3-selenadiazol-5-yl] sulfanyl-1-phenyl-1H-1,2,3,4-tetraazole (5b): $57 \%$ yield, m.p. $155-156^{\circ} \mathrm{C}$, time $1 \mathrm{~h} .{ }^{1} \mathrm{H}$ NMR $(300 \mathrm{MHz}$, DMSO-d $\left.\mathrm{d}_{6}\right): \delta 7.52-7.56(\mathrm{~m}, 5 \mathrm{H},-\mathrm{Ar}-H), 7.92(\mathrm{~d}, J=$ $8.6 \mathrm{~Hz}, 2 \mathrm{H},-\mathrm{Ar}-H), 8.26(\mathrm{~d}, J=8.6 \mathrm{~Hz}, 2 \mathrm{H},-\mathrm{Ar}-H)$; ${ }^{13} \mathrm{C}$ NMR (75 MHz, DMSO-d ${ }_{6}$ ): $\delta 124.7,125.4,130.7$, $130.9,131.8,132.2,135.3,146.4,148.1,159.8,170.3$. 
Anal. Calcd. for $\mathrm{C}_{15} \mathrm{H}_{9} \mathrm{~N}_{7} \mathrm{O}_{2} \mathrm{SSe}$ : C $41.87 \mathrm{H} 2.11 \mathrm{~N}$ 22.79. Found: C 41.99 H 2.18 N 22.84.

\subsection{General procedure for the preparation of 1,2,3-thiadiazole derivatives, $\mathbf{3 g}$ and $\mathbf{5 c}$}

Appropriate semicarbazone of $0.01 \mathrm{~mol}$ was added by portion to $10 \mathrm{ml}$ of thionyl chloride while cooling to $-5^{\circ} \mathrm{C}$ with a freezing mixture. The reaction mixture was allowed to stir for about $2 \mathrm{~h}$. The excess of thionyl chloride was decomposed using aqueous solution of sodium carbonate and extracted with chloroform. The product was obtained on purification by column chromatography using silica gel (60-120 mesh).

2.5a 4-(4-Chlorophenyl)-5-[(5-methyl-1,3,4-thiadiazol2-yl)sulfanyl]-1,2,3-thiadiazole (3g): $65 \%$ yield, viscous oil, time 1 h. ${ }^{1} \mathrm{H}$ NMR $\left(300 \mathrm{MHz}, \mathrm{CDCl}_{3}\right): \delta$ $2.82\left(\mathrm{~s}, 3 \mathrm{H},-\mathrm{CH}_{3}\right), 7.54(\mathrm{~d}, J=8.4 \mathrm{~Hz}, 2 \mathrm{H},-\mathrm{Ar}-H)$, $7.89(\mathrm{~d}, J=8.4 \mathrm{~Hz}, 2 \mathrm{H},-\mathrm{Ar}-H) ;{ }^{13} \mathrm{C}$ NMR $(75 \mathrm{MHz}$, $\left.\mathrm{CDCl}_{3}\right): \delta 15.9,128.5,129.3^{*}, 130.2,136.0,138.6$, $159.5,168.1$ (*One carbon merged with other). Anal. Calcd. for $\mathrm{C}_{11} \mathrm{H}_{7} \mathrm{ClN}_{4} \mathrm{~S}_{3}$ : C $40.42 \mathrm{H} 2.16 \mathrm{~N} 17.14$. Found: C 40.53 H 2.24 N 17.23.

$2.5 \mathrm{~b}$ 5-[4-(4-Chlorophenyl)-1,2,3-thiadiazol-5-yl] sulfanyl-1-phenyl-1H-1,2,3,4-tetraazole (5c): 69\% yield, m.p. $92-93^{\circ} \mathrm{C}$, time $2 \mathrm{~h} .{ }^{1} \mathrm{H}$ NMR $(300 \mathrm{MHz}$, $\left.\mathrm{CDCl}_{3}\right): \delta 7.44-7.63(\mathrm{~m}, 7 \mathrm{H},-\operatorname{Ar}-H), 7.34(\mathrm{~d}, J=$ $8.7 \mathrm{~Hz}, 2 \mathrm{H},-\mathrm{Ar}-H) ;{ }^{13} \mathrm{C} \mathrm{NMR}\left(75 \mathrm{MHz}, \mathrm{CDCl}_{3}\right): \delta$ $124.0,127.9,129.3,129.6,130.2,131.1,132.6,135.2$,
136.1, 149.8, 159.8. Anal. Calcd. for $\mathrm{C}_{15} \mathrm{H}_{9} \mathrm{ClN}_{6} \mathrm{~S}_{2}$ : C 48.32 H 2.43 N 22.54. Found: C 48.41 H 2.48 N 22.64.

\section{Results and discussion}

Heterocyclic compounds like 1,2,3- and 1,3,4thiadiazoles, tetarazole and 1,2,3-selenadiazole may assume more importance, if we link any two of these heterocyclic nuclei by a sulfur atom. The work described in this article deals with the synthesis of such type of compounds. Selenadiazole nuclei ${ }^{24-27}$ can be generated from semicarbozones with $\alpha$-methylene hydrogen by the reaction with selenium dioxide. Accordingly, appropriate semicarbazones have been synthesized to generate new selenadiazole ring. The semicarbazones $\mathbf{2 / 4}$ planned for this synthetic sequence can be obtained from the ketone $1 .^{28,29}$ The ketone $\mathbf{1}$ in turn can be prepared from appropriately substituted phenacyl bromide and 2-mercapto-5-methyl-1,3,4thiadiazole/1-phenyl- $1 H$ - tetrazole-5-thiol in presence of triethylamine. The substituted phenacyl bromides have been prepared by the reaction of the corresponding acetophenone with bromine in dry ether.

The semicarbazones $\mathbf{2 / 4}$ with $\alpha$-methylene hydrogens when treated with selenium dioxide undergoes oxidative cyclization to give 1,2,3-selenadiazole. The reaction is very much facile in acetic acid, tetrahydrofuran or ethanol. In the present case, we employed tetrahydrofuran as the solvent of choice. The semicarbazone and selenium dioxide was taken in tetrahydrofuran and heated on a water bath, the ratio of semicarbazone and selenium dioxide taken being 1:5. Upon heating for one hour, the reaction goes for completion

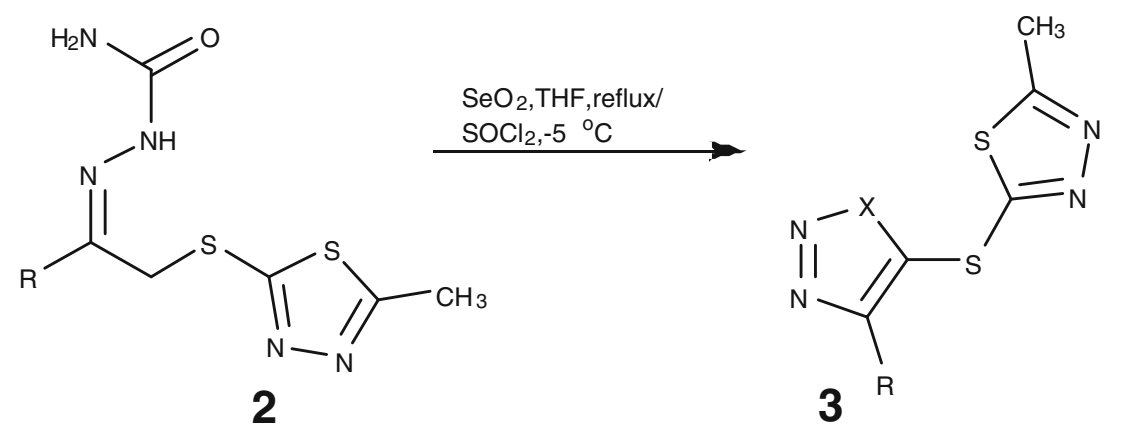

$$
\begin{aligned}
& \text { 2a : R=Phenyl } \\
& 2 \mathbf{b}: R=4-\text { Methylphenyl } \\
& \text { 2c : } R=4-\text { Methoxyphenyl } \\
& \text { 2d : } R=4-\text { Chlorophenyl } \\
& 2 \mathrm{e}: R=4-\text { Nitrophenyl } \\
& 2 \mathrm{f}: \mathrm{R}=2-\text { Naphthyl }
\end{aligned}
$$

Scheme 1. Synthesis of 1,2,3-selena/thiadiazoles derivatives, 3 . 


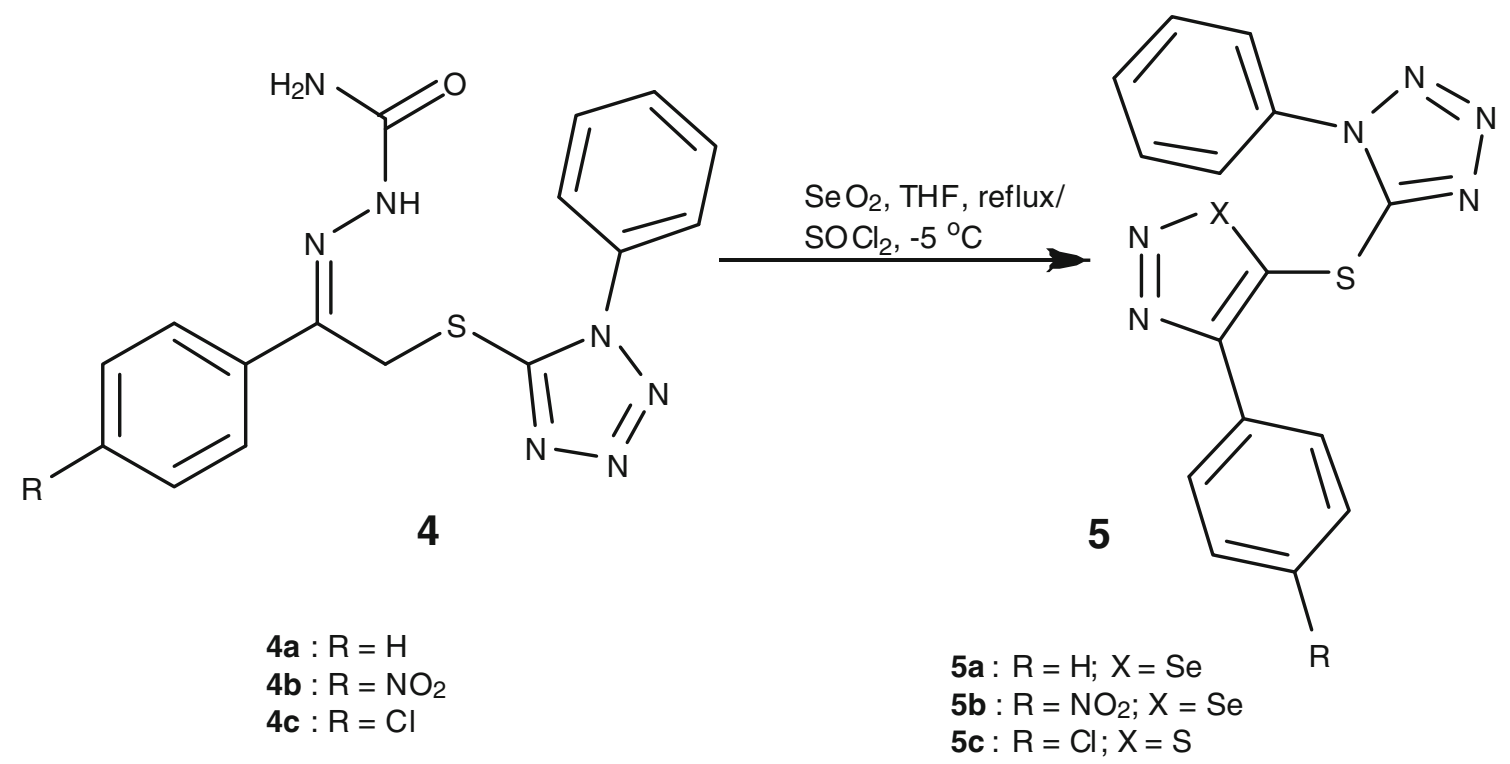

Scheme 2. Synthesis of 1,2,3-selena/thiadiazoles derivatives, 5 .

giving good yield of resultant selenadiazole $\mathbf{3 a - f}$ and 5a-b. (schemes 1 and 2).

The structure of the synthesized selenadiazole has been confirmed by ${ }^{1} \mathrm{H}$ and ${ }^{13} \mathrm{C}$ NMR spectra. The ${ }^{1} \mathrm{H}$ NMR spectrum of $\mathbf{3} \mathbf{c}$ has four signals, two doublets and two singlets at 7.66, 6.96, 3.77 and $2.69 \mathrm{ppm}$ respectively. The former two signals account for two hydrogens each, while the latter two signals account for three hydrogens each. In the ${ }^{13} \mathrm{C}$ NMR spectrum, the signal for the methyl carbon appears at $16.0 \mathrm{ppm}$, the signal for methoxy carbon appears at $55.4 \mathrm{ppm}$ and the methine carbons of the anisyl ring appear at 114.3 and $130.6 \mathrm{ppm}$. The quaternary carbons appear at 124.1, $140.6,158.3,159.8,160.3$ and $167.8 \mathrm{ppm}$. The HMBC spectrum helps to assign the different quaternary carbons. The methyl hydrogens at $2.69 \mathrm{ppm}$ has HMBC contour with carbon at $167.8 \mathrm{ppm}$ confirming this carbon to be the $5^{\text {th }}$ carbon of the thiadiazole ring. The methoxy hydrogens at $3.77 \mathrm{ppm}$ gives HMBC contour with the carbon at $160.3 \mathrm{ppm}$ helping to assign this carbon as the carbon ipso to methoxy. This carbon has HMBC contour with the hydrogens at $7.66 \mathrm{ppm}$ also. These hydrogen are the hydrogens meta to methoxy. These hydrogens give HMBC contour with carbon $159.8 \mathrm{ppm}$ and hence this carbon should be the carbon adjacent to nitrogen in the selenadiazole ring. The carbon para to methoxy can be easily assigned from the fact that the doublet at $6.96 \mathrm{ppm}$ gives a contour with the quaternary carbon at $124.1 \mathrm{ppm}$. The remaining two quaternary carbons are to be assigned between the carbons ipso to the sulfide ring.
After successfully generating selenadiazole rings attached to the 2-mercapto-5-methyl-1,3,4thiadiazole/1-phenyl-1H- tetrazole-5-thiol, we plan to prepare representative thiadiazole ring connected to tetrazole/thiadiazole with sulfur atom, as semicarbazones can also be subjected to cyclisation with thionyl chloride to give thiadiazole. The semicarbazones $\mathbf{2 d}$ and $\mathbf{4 c}$ were then treated with excess thionyl chloride at low temperature to give considerable yield of final products $\mathbf{3 g}$ and $\mathbf{5 c}$. Compounds $\mathbf{3 g}$ and $\mathbf{5} \mathbf{c}$ have been identified as the thiadiazole by ${ }^{1} \mathrm{H}$ and ${ }^{13} \mathrm{C}$ NMR spectra.

\section{Conclusion}

The synthesis of compounds in which two fivemembered heterocyclic compounds - each of them having at least three heteroatoms - are connected by a sulfur atom has been described.

\section{Supplementary information}

The NMR spectra of the synthesized compounds are provided in the supporting information and can be found at www.ias.ac.in/chemsci.

\section{Acknowledgement}

The authors thank the Department of Science and Technology (DST), New Delhi for the support under IRPHA for NMR facility. 


\section{References}

1. Kumar P, Mohan L J and Makrandi J K 2007 Indian J. Heterocycl. Chem. 1779

2. Kaur H, Sharma L M and Sangal S K 1994 Chimica Acta Turcica 22407

3. Santagati M, Modica M, Santagati A, Russo F, Caruso A, Cutuli V, Di Pietro E and Amico-Roxas M 1994 Pharmazie 49880

4. Kenned D A and Summers L A 1981 J. Heterocycl. Chem. 18409

5. Kishida K 1978 Chem. Pharma. Bull. 261049

6. Basavaraja K M, Somasekhar B and Appalaraju S 2008 Indian J. Heterocycl. Chem. 1869

7. Devi M A and Reddy V M 2008 Asian J. Chem. 205849

8. Song X-J and Tan X-H 2008 Phosphorus, Sulfur Silicon Relat. Elem. 1831755

9. Kuroda K, Uchikurohane T, Tajima S and Tsubata K 2000 U.S. Pat. 6166054 Chem. Abstr. 13438255

10. Fujii K, Hatano K and Yoshida Y 2001 Jpn. Kokai Tokkyo Koho 335570 Chem. Abstr. 13620074

11. Thomas E W, Nishizawa E E, Zimmermann D C and Williams D J 1985 J. Med. Chem. 28442

12. Jomaa H 2000 PCT Int Appl WO 0044359 Chem. Abstr. 133129848

13. Singh H, Chawla A S, Kapoor V K, Paul D and Malhotra R K 1980 Progress in medicinal chemistry, G P Ellis and G B West (eds); Elsevier/North-Holland, Biomedical Press 17151

14. Butler R N 1977 Adv. Heterocycl. Chem. 21323
15. Nash D T, Gross L, Haw W and Agre K 1968 Clin. Pharmacol. 8377

16. Jalilian A R, Sattari S, Bineshmarvasti M, Daneshtalab $M$ and Shafiee A 2003 Farmaco 5863

17. Mandour A H, El-Shihi V N, Abdel-Latif A and ElBazza Z E 1996 Phosphorus, Sulfur Silicon Relat. Elem. 113155

18. Kavitha C and Prasad K J R 2004 Asian J. Chem. 1640

19. Reddy D B, Reddy A S, Sekhar T C, Padmavathi V 1999 J. Ecotoxicol. Environ., Monit. 9225

20. Reddy D B, Reddy A S, Padmavathi V and Babu N C 2000 Heterocycl. Commun. 6271

21. El-Ezbawy S R, El-Kashef H S, El-Emary T A and Khalaf A A 1990 Bull. Fac. Sci. Assiut Univ. 1947

22. El-Kashef H S, Bayoumy B E and Aly T I 1986 Egypt. J. Pharm. Sci. 2727

23. Khanna P K 2005 Phosphorus, Sulfur Silicon Relat. Elem. 180951

24. Saravanan S and Muthusubramanian S 2004 Phosphorous, Sulfur Silicon Relat. Elem. 1792411

25. Saravanan S, Nithya A and Muthusubramanian S 2006 J. Heterocycl. Chem. 43149

26. Saravanan S, Athimoolam S and Muthusubramanian S 2007 Arkivoc i 22

27. Saravanan S, Muthusubramanian S, Vasantha S, Sivakolunthu S and Raghavaiah P 2007 J. Sulfur Chem. 28181

28. Lu Y and Zhang W 2006 QSAR Comb. Sci. 25728

29. Scifinder registry number 355222-66-7 307343-39-1 724757-18-0 677760-45-1 